\title{
Enhancing Energy Conservation Framework for Content Sharing On Smartphones
}

\author{
T.Priyanka ${ }^{1}$, P.Rajeswari ${ }^{2}$ \\ II-M.E(CS) $)^{1}$ H.O.D / ECE ${ }^{2}$, Dhanalakshmi Srinivasan Engineering College, Perambalur.
}

\begin{abstract}
Currently there is a plethora of social networks available and new ones are being introduced continuously. The information originate in these, range from information the users share to that generated from the interaction of the user with the system. Information from a single social network may be abundant enough to generate rich user profiles, which can be used for personalization services, recommendation services, etc. There is however an advantage in merging information from multiple information sources, to create richer user profiles. In this project we propose a peer to peer architecture to provide a fully decentralized system to generate and maintain distributed user models. Each peer in the system is made up of tribes for content replication among intended content consumers and serves it using low-cost network connections. Each peer in the system acts as a standalone user model; it extracts and translates information into pre-defined templates, which are used to ensure interoperability when communicating with other peers, through an internal server. A performance analysis for transmission rates and delays is therefore conducted. MobiTribe saves $76 \%$ of the uplink and $46 \%$ of the downlink cellular bandwidth compared to current widely used centralized server based content sharing architectures.
\end{abstract}

Keywords: peer-to-peer, content sharing

\section{Introduction:}

A social networking service is a platform to build social networks or social relations between peoples who, for example behavior, share happiness, backgrounds connections. A social network service consists of a illustration of every user, a variety of additional services and his/her social links. The device grouping algorithm identifies mTribe devices to maximize the availability of content via low-cost networks. The content replication process is managed by the CMS. Each device periodically (daily) updates the CMS with its binary Device Availability pattern, i.e. its ability to host others' content. The history of device availability patterns can be effectively used to infer future availability patterns of a device and groups of devices. The content will be uploaded to a centralized server at the time of sharing. With a centralized server, the shared content will be immediately available to the consumers. A distributed system is a software system in which components located on networked computers communicate and coordinate their actions by passing messages. The components interrelate with every other in order to achieve a common goal. Distributed decentralized social networking architectures that enable an individual user or a community of users to host data. Provide mechanisms that act upon local works, such as indirect contact, somewhat than the result of a central ordering power. Replication in computing involves sharing information so as to ensure consistency between unnecessary resources such as software or hardware components, to fault tolerance, improve consistency.

\section{Related Work:}

In [3] A. Balasubramanian, R. Mahajan, and A. Venkataramani et al. We then design a system, called Wiffer, to augments mobile 3G capacity. We design a system called Wi_er to overcome these availability and performance challenges. Wi_er leverages this observation to trade-off higher application latency for lower $3 \mathrm{G}$ usage. Wiffer quickly switches to $3 \mathrm{G}$ if $\mathrm{WiFi}$ is unable to transmit the packet within a time window. We implement and deploy Wiffer on a vehicular testbed. We evaluate Wiffer using the deployment and using tracedriven simulations. we find that Wiffer reduces $3 \mathrm{G}$ usage by $45 \%$ for a 60 second delay tolerance. Because of it's wait-only-if-it-helps strategy, the actual transfer latency is increased by only 7 seconds on average. Wiffer takes as input application data, which is characterized using S, the size of the transfer, D, the delay tolerance and an application-specified QoS metric. Based on these characteristics and those of the operating environment, it decides how to distribute the data across $3 \mathrm{G}$ and WiFi. To implement Wiffer's fast switching, we added a signaling mechanism in the mobile node's driver that signals the application when the wireless card receives a link layer acknowledgement.

In [4] N. Balasubramanian, A. Balasubramanian, and A. Venkataramani et al. we develop TailEnder, a protocol that reduces energy consumption of common mobile applications. we design TailEnder, an energyefficient protocol for scheduling data transfers. TailEnder considers two classes of applications: 1) delaytolerant applications such as email and RSS supply and 2) applications such as web search and web browsing 
that can benefit from aggressive prefetching. TailEnder determines what data to prefetch so as to minimize the overall energy consumed. TailEnder uses a probabilistic strategy to balance these concerns. Somewhat counterintuitively, for applications such as web search, TailEnder fetches several times more data and improves user-perceived response times, but still consumes less energy. We evaluate the performance of TailEnder for three different applications: email, news feeds and web search. We evaluate TailEnder by conducting experiments on the mobile phone and find that TailEnder can download 60\% more news feed updates and download search results for more than $50 \%$ of web queries, compared to using the default policy. We develop TailEnder, a protocol that minimizes energy usage while meeting delay-tolerance deadlines specified by users.

\section{Mobitribe}

Existing system allow scattered social networking on smartphones. PrPl is a service which allows a user to run the service on a home server. Safebook is stand on the idea of collaboration and decentralization between friends and produced a secure social network. Do not try to lower the contact cost and improved energy use. Accessibility is needy on the number of replicas. Existing system can direct to loss of confidentiality and user control. Mobitribe has skill in custom Mobile Application Development, Smart Phone Game Development and Smat Phone Application Development.

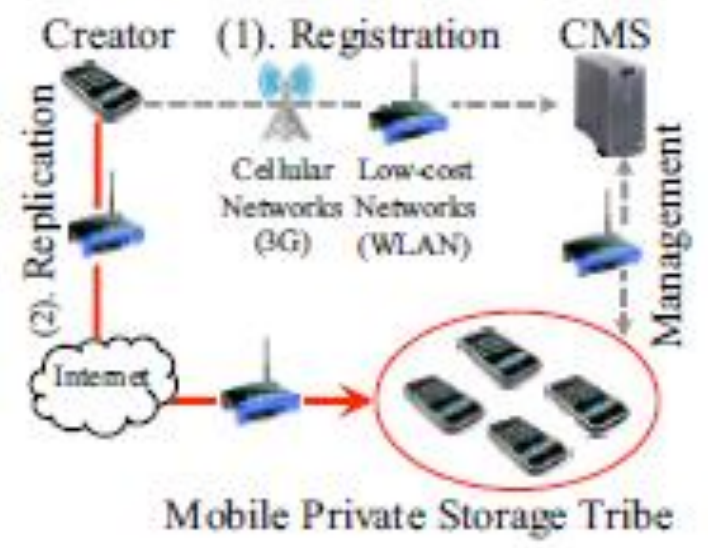

Figure 1: Content Sharing

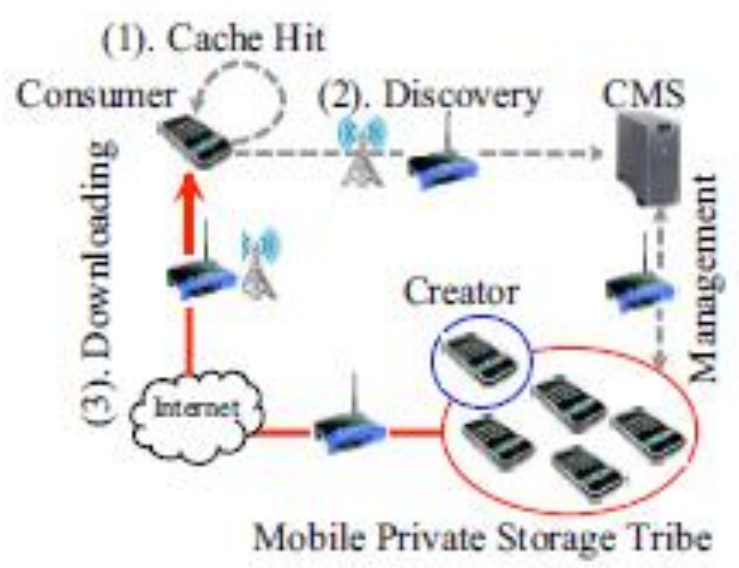

Figure 2: Content access process

We also concentrate in Graphics for Nokia Phone Mobile, Websites. We deploy, design \& develop successful applications for Smart Phone. Phone Apps, prepared for the different industries were utilized and functioning successfully all through Nokia Phone mobile users. We have specialist team who approach Nokia Phone application growth in a intended way. We organize with clients right from existing, We work with them throughout the Graphics Designing and Prototype for the Apps. Our idea growth process engages research and analysis on the range of the Mobile Application, its target users and its USP. 


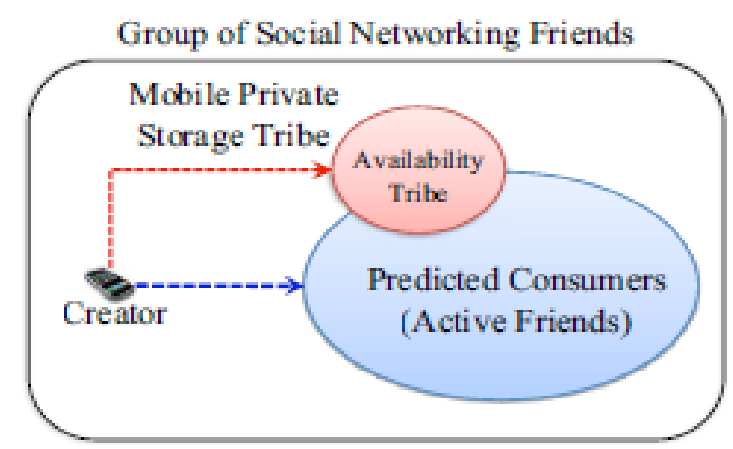

Figure 3: Replication tribe selection

\section{Load balancing based MobiTribe}

MobiTribe is a device centric social network using a "Connection Management Server" (CMS) that tracks the addresses and current connectivity of devices hosting content and manages the peer-to-peer content distribution. Sharing of content is done through registering the content with the CMS and saving its link, then replicating the content on a set of mobile devices called the "mobile private storage tribe". MobiTribe employs projecting pre-fetching of substance to minimize the overheads of content replication. As for accessing content, a content consumer initiates the content downloading process by clicking on a shared notification appearing in the social networking application.

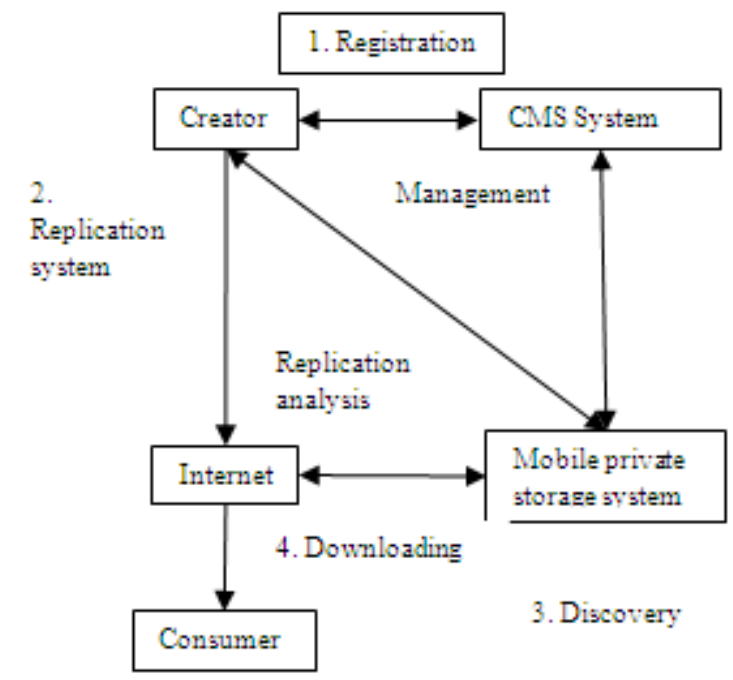

Figure 4: System Architecture

Preserve the privacy, while sharing contents in social networks. Each user device identifies potential shared content. Decrease the communication cost and save energy in our proposed work. MobiTribe utilizes the Facebook server to send the files registered at the CMS. So after deciding to share a torrent file, a media file is sent to the CMS. The CMS then returns a link to this file and shares it with online friends via the Facebook server. Since only links are stored at the CMS and Facebook is only used to get the list of user's friends and send them notifications, all data is kept away from service providers and transferred directly from one device to another.

\section{Experimental Results:}

Like all media sharing platforms, there is a certain overhead in order to share media between users. The extra data load is mainly for communication with the server in order to coordinate the sharing instances. However, the unstable local networks, mobile devices and behavior of users make it difficult to account for their effect; taking in mind that their overhead comes as delay, speed and reliability respectively. We will analyze the system behavior in each phase in order to generate equations specifying the variables in play at each step of operation. 


\begin{tabular}{|l|l|}
\hline Methods & Enery Consumption \\
\hline Mobitribe & 46 \\
\hline loadbalancing based mobitribe & 80 \\
\hline
\end{tabular}

Table 1: Comparison table

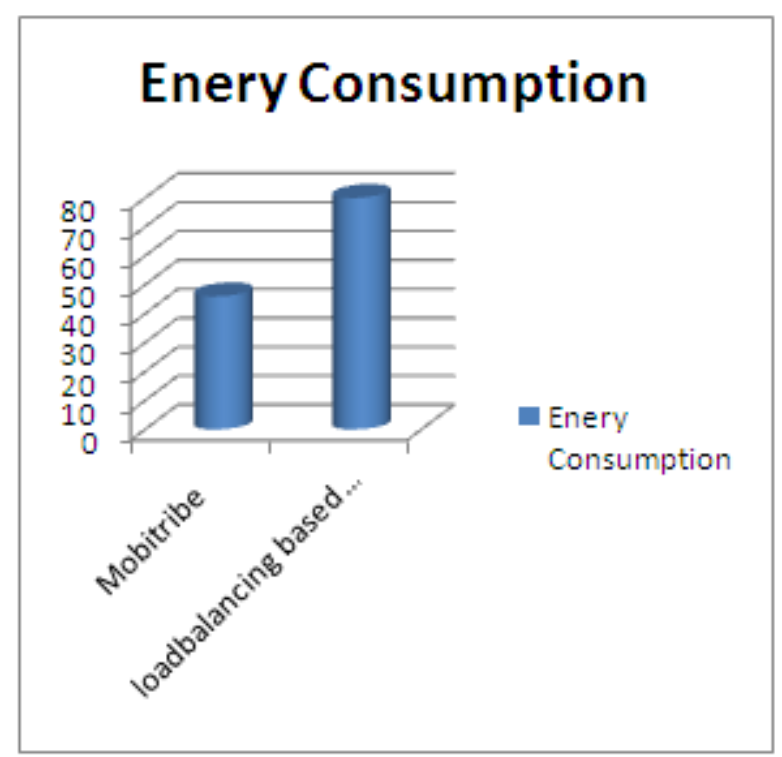

Figure 5: Comparison graph

\begin{tabular}{|l|l|l|}
\hline Methods & $\begin{array}{l}\text { Upload } \\
\text { Bandwidth }\end{array}$ & $\begin{array}{l}\text { Download } \\
\text { Bandwidth }\end{array}$ \\
\hline Mtribe & 234 & 543 \\
\hline Load Balancing Based Mtribe & 653 & 875 \\
\hline
\end{tabular}

Table 2: Comparison of Upload and download bandwidth

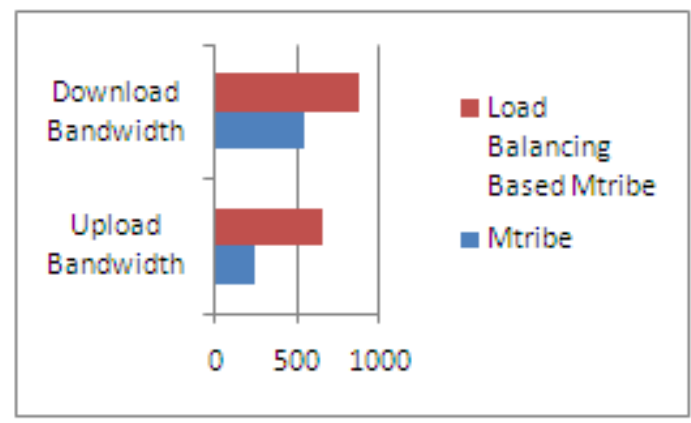

Figure 5: Comparison chart for upload and download bandwidth

\section{Conclusion:}

We proposed P2P architecture to generate and maintain a distributed user model, which uses predefined information exchange templates to communicate the user model. The provision condition and exchange is controlled by standalone peers who contain the necessary logic to process, and store the information from a single information source. Each peer is self contain and can proceed as standalone user model which only handles information from a single source. The objective however is to leverage information from numerous sources, with no requires handling the workload of extracting, storing and processing this information individually. A peer not only extracts data, but it also convert it into pre-determined templates, in order to communicate it with other peers in the system. A peer can act either as a consumer, a producer or both, depending on its preferred effect on the entire system. Adding data replication to the system, i.e. automatically sending the data from the host to one of the recipients, will help with increasing the availability of the data. Thus, even if the host goes offline after posting media, the data will still be available at the selected recipient. We can extend our work to analyze content replication using load balancing algorithm to checks the status of users and reduce false positive prediction in social networks. 


\section{References:}

[1]. Crawdad [Online]. Available: http://crawdad.cs.dartmouth.edu

[2]. A. Abhari and M. Soraya, "Workload generation for youtube,” Multimed. Tools Appl., vol. 46, no. 1, pp. 91-118, Jan. 2010.

[3]. A. Balasubramanian, R. Mahajan, and A. Venkataramani, "Augmenting mobile 3G using WiFi," in Proc. MobiSys, San Francisco, CA, USA, 2010, pp. 209-222.

[4]. N. Balasubramanian, A. Balasubramanian, and A. Venkataramani, "Energy consumption in mobile phones: A measurement study and implications for network applications," in Proc. 9th ACM SIGCOMM IMC 2009, Chicago, IL, USA, pp. $280-293$.

[5]. Citrix ByteMobile. (2011, Oct.). Mobile Analytics Report [Online]. Available: http://www.bytemobile.com

[6]. G. Calinescu, C. Chekuri, M. Pál, and J. Vondrák, "Maximizing a monotone submodular function subject to a Matroid constraint," SIAM J. Comput., vol. 40, no. 6, pp. 1740-1766, 2011.

[7]. M. Cha, H. Kwak, P. Rodriguez, Y. Ahn, and S. Moon, “Analyzing the video popularity characteristics of large-scale user generated content systems,” IEEE/ACM Trans. Netw., vol. 17, no. 5, pp. 1357-1370, Oct. 2009.

[8]. "Visual networking index: Usage," Cisco Systems, Inc., White Paper: FLGD 09319, Oct. 2010.

[9]. "Visual Networking Index: Global mobile data traffic forecast update 2012-2017," Cisco Systems, Inc., White Paper: FLGD 10855, Feb. 2013

[10]. M. Cunche, M.-A. Kaafar, and R. Boreli, "I know who you will meet this evening! Linking wireless devices using Wi-Fi probe requests," in Proc. IEEE Int. Symp. WoWMoM, San Francisco, CA, USA, Jun. 2012, pp. 1-9. 